\title{
Annual Meeting Theme for 1992: Developmental Neurobiology and Clinical Course
}

Four salient features of pathophysiology receive inadequate attention in biological psychiatry. First, most psychiatric disorders have relatively characteristic ages of onset. Second, most have a lifetime course. Third, the clinical phenomenology associated with a given diagnosis tends to change as the person ages. Fourth, gender and reproductive milestones strongly influence clinical onset, features and course. These observations suggest that neurobiological changes in the brain associated with aging and development are profoundly important in understanding pathophysiology and clinical presentation of psychiatric disorders. What are these neurobiological changes? When and where in the brain do they occur? Can they be predicted? Recognized clinically? Identified by laboratory tests? Altered by treatment? Sadly, many of these questions cannot be answered, since we know too little about developmental neurobiology and clinical course. This void exists despite the fact that longitudinal course may be the most inportant aspect of clinical psychiatry.

In a small attempt to fill this void and stimulate future investigation, the 1992 Scientific Meeting of the Society of Biological Psychiatry (in Washington, DC, April 29-May 3) wil! highlight the theme "Developmental Neurobiology and Clinical Course."

As background, let us review briefly the evidence supporting the above observations. Some entities such as Tourette's disorder, attention deficit disorder, and autism usually appear during childhood. Others-schizophrenia and panic disorder are examples-have onsets predominantly during childhood, adolescence and young adulthood. Some can present at any age, but with incidence progressively increasing as years go by; major depression, once erroneously believed to be a disease of middle age is an example. Still other syndromes, such as Alzheimer's dementia, seemingly occur only in the aging brain, almost never appearing at a young age (although the brains of patients with Down's Syndrome have neuropathological features in common with Alzheimer's). For certain pathophysiologies, there clearly are high risk periods followed by a lessening of risk if the hazardous period is safely passed. For other disorders there is a progressive increase of risk with each passing year. Expanding our knowledge base about the mechanisms underlying these risk milestones may tell us a great deal about the etiology of many disorders.

Regarding longitudinal course, most psychiatric disorders, once developed, do not go away permanently. Some are episodic and recurrent, others chronic and stable, still others chronic and deteriorating; but sadly, most persist over the life cycle. Lifetime course is so characteristic for some disorders that it may be a more powerful predictor of diagnosis than are clinical features. For example, if a clinician were informed that a patient had initial onset of clinical symptoms after childbirth, good short-term resolution of symptoms, five or six similar episodes over the next two decades, with episodes getting closer together as the years passed, even if that clinician were never aware of a single clinical symptom, he or she would have an extremely high likelihood of accurately diagnosing an affective 
disorder. What neurobiological network induces this episodic, recurrent course in the brain of some depressives? How many patients have it? Are aging and the number of depressive episodes interactive in its production? Is the pattern consistent in family cohorts? Does its presence have predictive importance in planning maintenance treatment? This litany of important but largely unanswered questions could be repeated for most psychiatric disorders included in DSM-III-R.

The third observation addresses clinical phenomenology across the life cycle. Considerable data suggest that clinical features for a given diagnosis are not the same at all ages. An 80 year old with depression generally presents with a different constellation than an eight year old, with the elderly person being more likely to manifest agitation, hyposomnia, hypophagia and psychosis. Depression is not necessarily identical at all ages. Neither are most other diagnoses. Why?

The final observation-that gender and reproductive milestones profoundly influence clinical onset, features and course-also is amply supported by clinical experience. Some disorders, such as anorexia nervosa, bulimia, and borderline personality disorder tend to be almost exclusively associated with women; others, such as Tourette's, alcoholism and sociopathy are preponderant in men. Puberty induces a major shift in the sex ratio of depressed children, from a male preponderance before puberty to a female preponderance after puberty. Certain pathophysiologies may appear or be accentuated only at certain times in the reproductive cycle. Again, for each of these observations, why? What are the underlying mechanisms?

Longitudinal patterns are important. They are relevant to diagnosis and prognosis. They have made the development of laboratory tests more difficult, since "normal" results often change with aging. Perhaps most importantly, different longitudinal patterns probably have major treatment implications. Emerging new data, especially for mood disorders, provide powerful support for the long-held belief that for many patients, maintenance psychopharmacological treatment should receive more attention than short-term interventions. Rather than being preoccupied with the question of when medications can be discontinued, clinicians perhaps should give more attention to the question of which patients require ongoing medication, often including lifetime medication.

If developmental neurobiology across the life cycle is so relevant, why has it been relatively neglected since the days of Kraepelin? There are a number of identifiable reasons. First, psychiatry's tradition of emphasizing "inpatient" and "outpatient" delivery systems has impeded long-term perspectives. Second, DSM-III has unduly prioritized cross-sectional assessment of clinical features for diagnosis, often neglecting longitudinal course in the process. Third, research funding as a catalyst generally has been lacking; longitudinal, long-term studies are difficult, costly, and time-consuming, and funding priorities in our institutes have rarely taken long-term perspectives. Fourth, promotion and tenure policies in most universities pressure junior academicians to develop shortterm studies capable of producing publishable results sooner rather than later. Fifth, and perhaps most discouragingly, since it is still gaining momentum, psychiatry's penchant for age-oriented subspecialization is a barrier to better understanding of longitudinal course. Our traditional breakdown into child, adolescent and adult subspecialties has interfered with optimal scientific exchange (perhaps explaining why depression was not even recognized in children and adolescents until several decades ago); now, infant and geriatric subspecialties will further fragment attempts to develop a perspective on the complete "ages of man." This subspecialty barrier will be difficult to overcome since different age-oriented subspecialists generally don't practice in the same settings, don't 
see the same patients to compare inter-rater reliability, don't attend the same meetings, don't talk with subspecialists who emphasize different ages, don't always talk the same professional language, and don't even have the same accreditation and regulatory agencies. The other barriers will be just as difficult to break down. But we must try.

In this "Decade of the Brain," it is time for biological psychiatry to rediscover developmental neurobiology and longitudinal course, to reacquaint ourselves with the considerable knowledge we have compiled through the years, and to formulate research priorities for the future. This 1992 Scientific Meeting will strive to aid these initiatives. We look for your assistance. I am pleased to represent the officers of the Society in welcoming you to Washington, D.C. from April 29-May 3, 1992. It should be an excellent meeting.

Department of Psychiatry

John F. Greden

President, Society of Biological Psychiatry

University of Michigan

1500 East Medical Center Drive

Ann Arbor, MI 48109-0704 